\title{
ARTICLE
}

\section{BioAIEgens Derived from Rosin: How Does Molecular Motion Affect Their Photophysical Processes in Solid State?}

\author{
Xu-Min Cai ${ }^{1,2,4}$, Yuting Linn ${ }^{1,4}$, Ying $\mathrm{Li}^{2,4}$, Xinfei Chen ${ }^{1}$, Zaiyu Wang ${ }^{2}$, Xueqian Zhao ${ }^{2}$, Shenlin \\ Huang ${ }^{1}$, Zheng Zhao ${ }^{2,3}$, \& Ben Zhong Tang ${ }^{2}$
}

\begin{abstract}
The exploration of artificial luminogens with bright emission has been fully developed with the advancement of synthetic chemistry. However, many of them face problems like weakened emission in the aggregated state as well as poor renewability and sustainability. Therefore, the development of renewable and sustainable luminogens with anti-quenching function in the solid state, as well as to unveil the key factors that influence their luminescence behavior become highly significant. Herein, a new class of natural rosinderived luminogens with aggregation-induced emission property (AIEgens) have been facilely obtained with good biocompatibility and targeted organelle imaging capability as well as photochromic behavior in the solid state. Mechanistic study indicates that the introduction of the alicyclic moiety helps suppress the excited-state molecular motion to enhance the solid-state emission. The current work fundamentally elucidates the role of alicyclic moiety in luminogen design and practically demonstrates a new source to largescalely obtain biocompatible AIEgens.
\end{abstract}

\footnotetext{
${ }^{1}$ Jiangsu Co-Innovation Center of Efficient Processing and Utilization of Forest Rescources, College of Chemical Engineering, Nanjing Forestry University, Nanjing 210037, China. ${ }^{2}$ Department of Chemistry, Hong Kong Branch of Chinese National Engineering Research Center for Tissue Restoration and Reconstruction, Institute of Molecular Functional Materials, Division of Life Science and State Key Laboratory of Molecular Neuroscience, The Hong Kong University of Science and Technology, Clear Water Bay, Kowloon, Hong Kong 999077, China. ${ }^{3}$ School of Chemistry and Engineering, Southeast University, Nanjing 211189, China. ${ }^{4}$ These authors contributed equally: Xu-Min Cai, Yuting Lin, and Ying Li. Correspondence and requests formaterials should be addressed to S.H. (email: shuang@njfu.edu.cn) or to Z.Z. (email:zhaozheng@seu.edu.cn) or to B.Z.T. (email: tangbenz@ust.hk)
} 
Light has played an irreplaceable role in the long history of human development. Besides illumination, light has been applied in many advanced technologies, including optoelectronics, fiberoptic communication as well as optical diagnosis. ${ }^{1-3}$ As the source of light, the earliest record of luminescent substances was an infusion of a Mexico wood with blue emission, which can be dated back to the $16^{\text {th }}$ century. ${ }^{4-6}$ Since then, luminescent matters had attracted people's attention and various luminescent matters such as inorganic stones and natural products or organism were gradually discovered and investigated..$^{7-9}$ However, due to the limitations in variety and difficulty in the study of structure-property relationship, the understanding on luminescence phenomenon and the development of luminescent materials is generally lagged. Until the fast development of synthesis technology, facile acquirement of artificial luminescent materials as well as systematical study on the structure-property relationship could be achieved. ${ }^{10-11}$ Regretfully, the aggregation-caused quenching (ACQ) phenomenon always occurs, prohibiting its application of luminescent materials in high concentration or aggregated state. ${ }^{12-14}$ Therefore, how to address this ACQ problem has become one of the most flourishing research topics in recent decades.

Luminogens with aggregation-induced emission (AIE) has aroused intensive interests since this kind of materials possesses unique characteristic distinguished from that of $\mathrm{ACQ}$ ones. ${ }^{15}$ Thanks to their aggregate-state emission and aggregationresponsive luminescence, AIE luminogens (AIEgens) have exhibited promising applications in organic optoelectronics, stimuli-responsive materials, chemo-/bio- sensors, and luminescent theranostics. ${ }^{16-21}$ Thousands of new AlEgens thus have been designed and synthesized, most of which were structurally designed on conjugated moieties. However, many of their preparation procedures require complicated synthesis and purification techniques, making them uneasy to be obtained, let alone their abundant pollution hazards. Alternatively, plantoriginated chemicals are green and bioactive compounds that have been widely studied as one of the most interesting naturebased compounds in the advanced (bio)sensing fields because of their numerous inherent and unique characteristics, such as natural availability, renewability, sustainability, biodegradability, and biocompatibility. ${ }^{22}$ Hence, AlEgens from natural resources have recently gained researchers' intense attention (Figure S1). ${ }^{23-}$ 32 To date, some natural product-based AIEgens including natural polymers (sodium alginate and bovine serum albumin) and small molecules (quercetin, myricetin, and berberine chloride) have been investigated with unique advantages in bioapplications. Due to their advantageous biocompatibility, imaging can be applied either in vitro or in vivo. Nevertheless, in general, the development of these bioproduct-inspired AlEgens (BioAIEgens: AlEgens or AIE-active nano agents obtained from natural resources including natural products or derivatives by modifying natural products) ${ }^{21}$ is still in its infant and the limited variety of AIEgens from natural resources hinders their further investigation and development. In consideration of these, BioAlEgens derived from the chemical modification on the abundant and low-cost natural resources might be a good alternate to either pure natural or artificial AlEgens.

Natural rosin can be easily obtained from pine resins and some other plants, which has been used as a cheap feedstock chemical to replace petrochemicals in industry. ${ }^{33}$ It is mainly composed of two types of rosin acids: abietic and pimaric acid. Among them, dehydroabietic acid (DA) belongs to the type of abietic acid, which is the largest and most stable component. ${ }^{34-35}$ To increase the additional value of DA-based chemical products, studies on DA derivatives and their applications have been extensively carried out, of which the pharmaceutical research is under specific focus. ${ }^{36-41}$ However, their application in optical functional materials have been scarcely investigated. From the structural point of view, the aniline part of DAMBA (a derivative of $D A$; vide infra) could be easily coupled with salicylaldehyde to yield Schiff bases, which usually owns flexible structure with photoisomerization property. ${ }^{42-45}$ While the alicyclic (decalin) structure of DAMBA actually could be thought as a structural analogue to phytosterol and cholesterol, which has been commonly used by plant and zooblast cells to stiffen the unsaturated lipid membrane and sustain the normal physiological functions. ${ }^{46}$ Hence, structural marriage of the flexible Schiff bases with the rigid decalin structure of DAMBA potentially provides a strategy to regulate the excited-state molecular motion of Schiff bases and endows the generated molecules with unexpected photophysical properties or functions. Noteworthy, the luminescent properties correlate closely with their excited-state molecular motions, which unfortunately occurs in an ultrafast timescale that is too fast to trace and regulate. The fully understanding and regulation of the molecular motions on the excited state is thus of great significance for the rational and accurate design of new luminescent materials. ${ }^{47-51}$

Herein, we have succeeded a series of alicycle-fused Schiff bases as a new class of BioAlEgens derived from natural rosin. The alicyclic moieties help to rigidify the flexible structure of the non-emissive Schiff base to light up its fluorescence. Furthermore, the luminescence properties could be facilely tuned by structural substitution since the substitution variations could easily affect their intermolecular interactions and their excited-state molecular motion. Such molecular motion distinction-resulted luminescence variation could also be monitored and visualized in the solid state by either fluorescence or photochromic properties. Finally, due to the lipid characteristic and biocompatibilities of these BioAIEgens, they were applied in cell imaging, exhibiting superior organelle selectivity in lipid droplet and lysosome, respectively. This work not only demonstrates a new renewable and sustainable source to acquire BioAIEgens and unveils the underlying structureproperty relationship, but also provides a new rigidification strategy to improve the efficiency of luminogens.

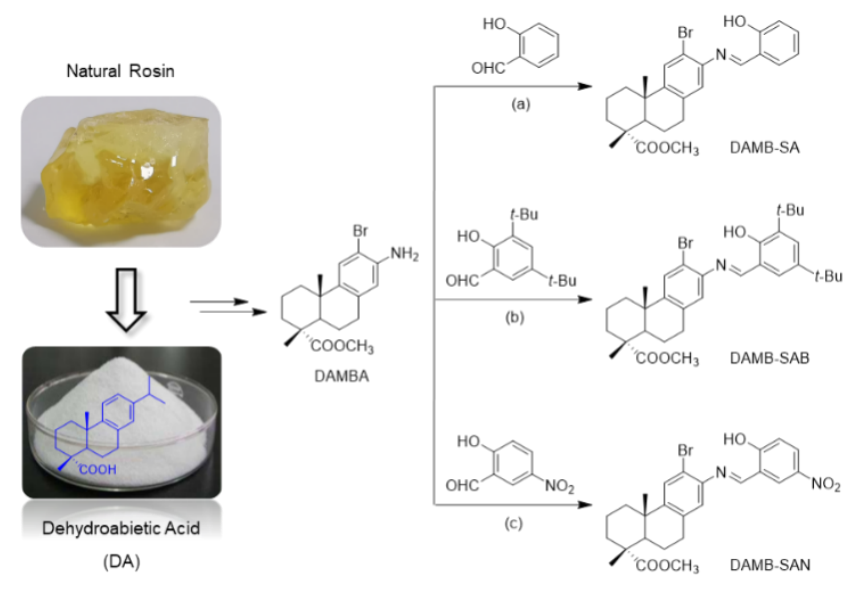

Fig. 1 Synthesis of dehydroabietic acid-derived Schiff bases: (a) $\mathrm{EtOH}, 90^{\circ} \mathrm{C}$ $10 \mathrm{~h}$; (b) $\mathrm{EtOH}, 100^{\circ} \mathrm{C}$, over night; (c) $\mathrm{EtOH}, 85^{\circ} \mathrm{C}, 3 \mathrm{~h}$. 


\section{Results and discussion}

Molecular synthesis and characterization. As shown in Fig. 1, DA extracted from disproportionated rosin could be chemically modified to its derivative DAMBA ${ }^{41}$ which is herein applied as a precursor to react with a series of various substituted salicylaldehyde to yield their corresponding Schiff bases, DAMBSA, DAMB-SAB, and DAMB-SAN, with a high crystalline yield of around $70 \%$ without chromatographic purification. The structures and purity of the obtained products were confirmed by melting point, NMR as well as high resolution mass spectroscopy measurements (Fig. S2-S11). Single crystals of all Schiff bases suitable for X-ray diffraction measurement could be easily obtained via slow evaporation in their respective solutions (please see the details in the Methods).

Aggregation-induced emission properties. Fig. $2 \mathrm{~A}$ gives us an intuitive impression about the fluorescence properties of these alicycle-fused Schiff bases (Fig. 2B-D and S12). DAMB-SA and DAMB-SAB show no emission in acetonitrile $(A C N)$ solutions but enhanced yellow and dull orange emissions when adding water into the ACN solutions, suggesting their typical AIE characteristic. As a comparison, DAMB-SAN is emissive in both the pure ACN solution and aggregates ( $f_{w}=90 \%$ ), with green and greenish yellow light, respectively. DAMB-SAN thus shows aggregationenhanced emission property. Dynamic light scattering (DLS) results (Fig. S13) support that nano aggregates of all DA-derived Schiff bases are formed at high $f_{w}(80 \%)$, and their enhanced emission in $\mathrm{ACN} / \mathrm{H}_{2} \mathrm{O}$ mixtures is derived from their aggregation in poor solvents. Furthermore, concentration experiment also supports the AIE characteristic of these alicycle-fused luminogens since much enhanced emission can be observed at a higher concentration (Fig. S14). The discernible fluorescence colors inform us that the substituent variations must have played a critical role. Indeed, DFT calculation indicates that the substitution groups cause different band gaps of DAMB-SAB (3.87 eV), DAMB-SA (3.96 eV) and DAMB-SAN (3.98 eV), which matches well with their luminescence color change trend (Fig. S15). Additionally, no solvent effect can be found in the solvatochromic tests of DAMB-SAN (Fig. S16), indicating that charge transfer might not be the main reason to the emission wavelength variations. Besides their fluorescence color, the luminescence efficiency of the DA-derived AlEgens also shows obvious difference with solid-state quantum yields (QYs determined by an integrating sphere) of $1.5 \%, 0.3 \%$ and $4.9 \%$ for DAMB-SA, DAMB-SAB, and DAMB-SAN, respectively. According to the AIE mechanism of restriction of intramolecular motion (RIM), the luminescence efficiency relates closely with the excited-state molecular motion that active excited-state molecular motion normally promotes the non-radiative decay channel to decrease the luminescence efficiency. Since DAMB-SA, DAMB-SAB, and DAMB-SAN share the same fundamental chromophoric structure of $A B-S A$, it is rational to compare the luminescence properties of the alicycle-fused AIEgens with their respective control molecules of $A B-S A, A B-S A B$, and $A B-S A N$ to deeply understand their luminescence behaviors. Interestingly, although these alicyclefused AIEgens are all AIE active with enhanced emission in the solid state (taking DAMB-SA as an example, Fig. 2E), AB-SA is surprisingly non-luminescent either at molecular or aggregate level (Fig. 2F). In addition, although the solid state of AB-SA is non-fluorescent at room temperature, it emits very strongly at 77 $\mathrm{K}$. These results suggest that AB-SA has active excited-state molecular motion even in the solid state, which quenches its emission. While only in the ultralow temperature of $77 \mathrm{~K}$, the molecular motion could be suppressed to generate emission. Furthermore, AB-SAB and AB-SAN exhibit weaker emission
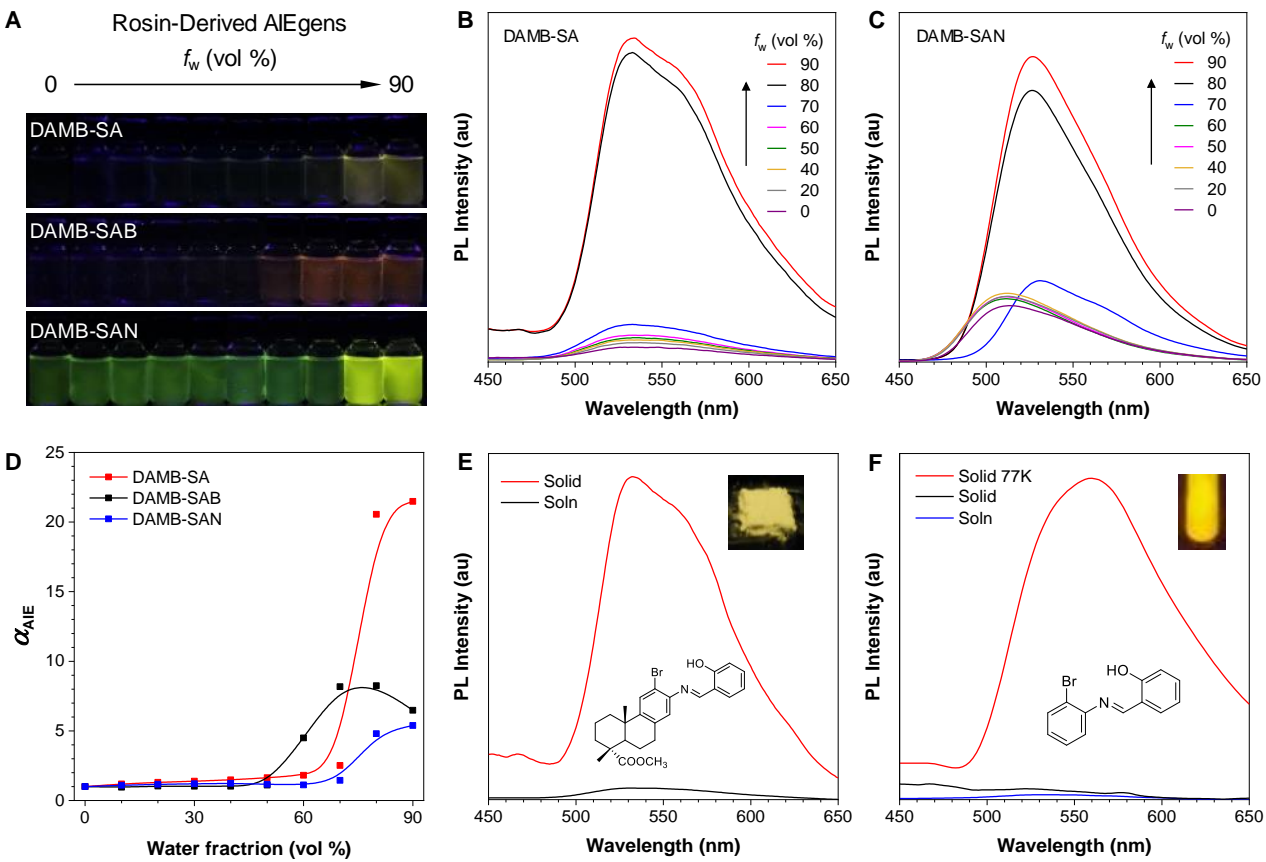

Fig. 2 (A) Fluorescence photographs of DAMB-SA, DAMB-SAB, and DAMB-SAN in ACN/ $\mathrm{H}_{2} \mathrm{O}$ mixtures with different water fractions $\left(f_{w}\right)$ taken under 365 nm UV irradiation. Concentration: $20 \mu \mathrm{M}$. (B and C) PL spectra of DAMB-SA (B) and DAMB-SAN (C) in ACN/ $\mathrm{H}_{2} \mathrm{O}$ mixtures with different $f_{w}$. Concentration: $20 \mu \mathrm{M}$; $\lambda_{\text {ex: }} 350$ $\mathrm{nm}$. (D) The plots of the $\alpha_{\mathrm{AIE}}$ versus the composition of the aqueous mixture of DAMB-SA, DAMB-SAB, and DAMN-SAN. $\alpha_{\mathrm{AIE}}=I / I_{0}, I_{0}=\mathrm{PL}$ intensity in pure ACN (E) PL spectra of DAMB-SA in dilute ACN solution $(20 \mu \mathrm{M})$ and as solid at room temperature, respectively. (F) PL spectra of AB-SA in dilute ACN solution (20 $\mu \mathrm{M})$ and as solid at room temperature and $77 \mathrm{~K}$, respectively. $\lambda_{\mathrm{ex}}$ : $350 \mathrm{~nm}$. Inset: fluorescence photographs of DAMB-SA (room temperature) and AB-SA ( $77 \mathrm{~K}$ ) taken under $365 \mathrm{~nm}$ UV irradiation. Concentration: $20 \mu \mathrm{M}$. 


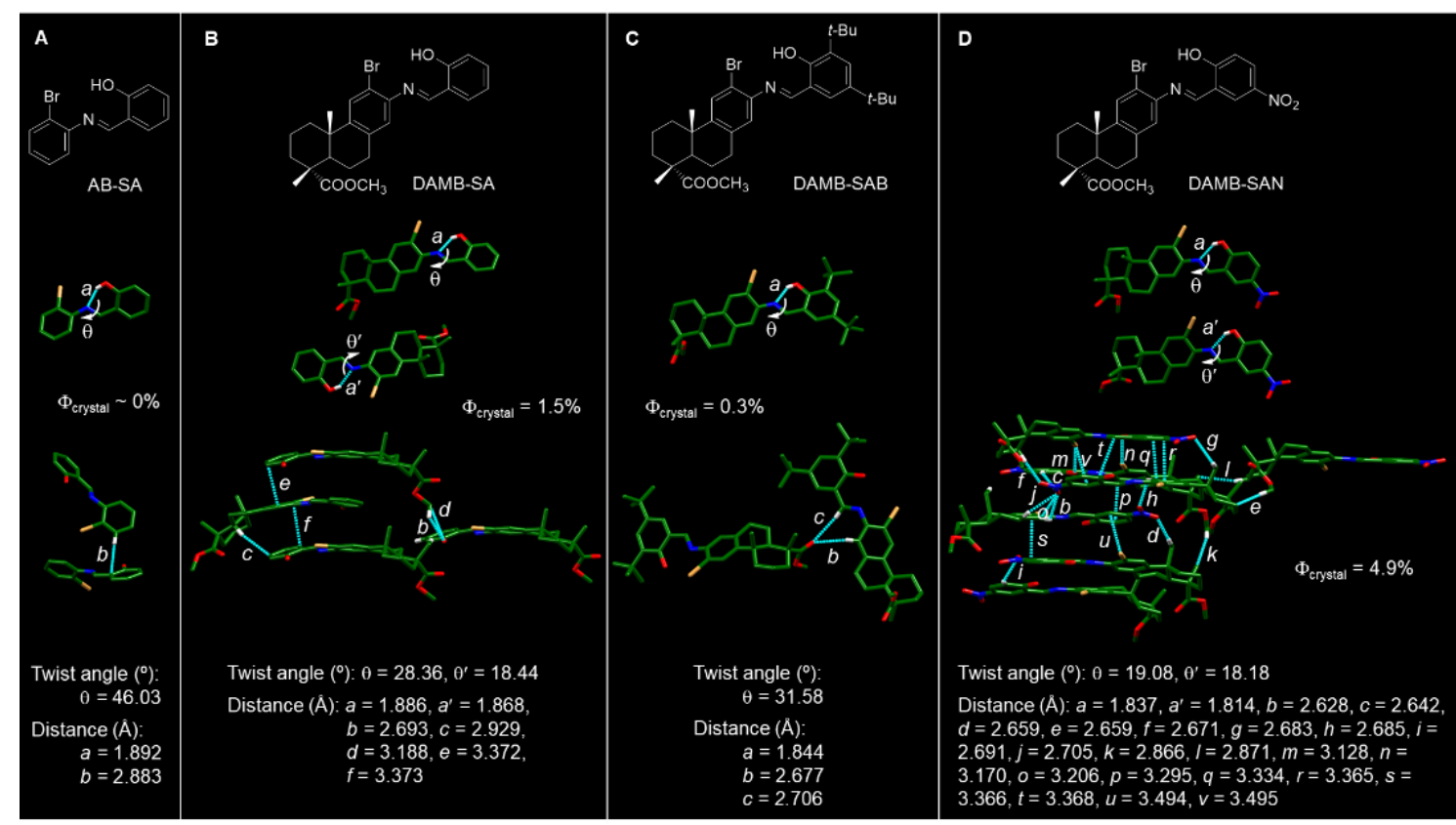

Fig. 3 Chemical structures (top), single crystal structures (middle), and their respective intermolecular interactions (bottom) of (A) AB-SA, (B) DAMB-SA, (C) DAMB $\mathrm{SAB}$, and (D) DAMB-SAN.

efficiencies than DAMB-SAB and DAMB-SAN (Fig. S17; QYs for AB-SAB, AB-SAN, DAMB-SAB, and DAMB-SAN: $0.1 \%, 0.2 \%$, $0.3 \%$, and $4.9 \%$ ). Based on the above observation and further comparing the luminescence of control molecules with the alicycle-fused AIEgens, it is rational to speculate that the alicyclic moiety in the DA skeleton may help to rigidify the molecular conformation to result in the AIE property of these alicycle-fused AlEgens. However, the conformational rigidification effect of the alicyclic moiety on varied substituted AB-SA structures are also different, resulting in their variation in luminescence efficiency.

Single crystal structure and crystal packing analysis. In order to shed more light on the cause of their fluorescence distinctions and confirm our speculation, all alicycle-fused BioAlEgens together with the basic structure of AB-SA have been crystallographically measured and analyzed (Fig. 3 and Table S1). Among all, $A B-S A$ has the more twisted structure and larger

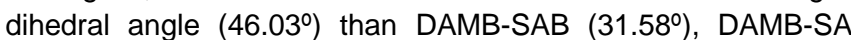
(28.36 and $\left.18.44^{\circ}\right)$, and DAMB-SAN (19.08 and 18.18 ${ }^{\circ}$ ). More twisted molecular structure is unfavorable to stabilize the excitedstate molecular conformation through electron delocalization and resonance. As a comparison, DAMB-SAN possesses the flattest conformation that helps to stabilize the excited molecular conformation, resulting in its luminescence behavior in solution state. Noteworthy, the intermolecular interactions of DAMB-SA, DAMB-SAB, and DAMB-SAN in the crystal state also show interesting changes relevant to AB-SA. Overall, AB-SA exhibits the least intermolecular interactions with a relatively loose molecular arrangement and weak intermolecular restriction effect. This kind of molecular arrangement benefits the excited-state molecular motion and thus can well explain the non-luminescence behavior of AB-SA in both solution and crystal state. However, when the alicyclic unit is fused on the molecular backbone, the intermolecular interaction becomes stronger which has increased the restrain effect of the crystal arrangement of the BioAlEgens.
And the restrain effect exhibits a proportional effect to the luminescence efficiency of these BioAIEgens. For example, according to the crystal arrangements in Fig. 3, the intermolecular interactions of DAMB-SAB, DAMB-SA, and DAMB-SAN exhibit a gradual increase tendency, which is in good consistence with their luminescence efficiency change (QYS of DAMB-SAB: $0.3 \%$, DAMB-SA: $1.5 \%$, and DAMB-SAN: $4.9 \%$ ). What's more, the melting point tendency (DAMB-SAN: $216-217{ }^{\circ} \mathrm{C}$, DAMB-SA 156-157 ${ }^{\circ} \mathrm{C}$, DAMB-SAB: $104-105{ }^{\circ} \mathrm{C}$, and AB-SA: $85-86{ }^{\circ} \mathrm{C}$ ) matches well with their intermolecular interaction degree from DAMB-SAN, DAMB-SA, DAMB-SAB to AB-SA. Hence, it is rational to conclude that the alicyclic moiety together with the substituent groups endow AB-SA either a better conjugation at molecular state or enhanced intermolecular interactions at aggregate state, resulting in the more restrained excited-state molecular motion and the AIE behavior. In order to further verify the role of molecular motion in determining their fluorescence properties, we took DAMB-SA as an example and studied the fluorescence changes upon its viscosity and temperature variations. As shown in Fig. S18, when glycerol is added to the DAMB-SA solution in an $\mathrm{EtOH} /$ glycerol mixture to increase its viscosity, the emission becomes stronger due to the suppression of the molecular motion. Similar to the viscosity variation, the emission of the DAMB-SA solution in ACN at $77 \mathrm{~K}$ is extremely strong while that at room temperature is totally non-fluorescent (Fig. S19).

Visualization of photochromic procedures. Simultaneous regulation and visualization of the solid-state molecular motion is a challenge although it is significant to understand the structural design of molecular machines or photo-responsive smart materials. ${ }^{47-51}$ Herein, the active molecular motion of AB-SA and the conformational rigidification effect of alicyclic moiety enable the regulation of the solid-state molecular motion of the generated BioAIEgens. Superior to the monitoring of structural variation from 
A

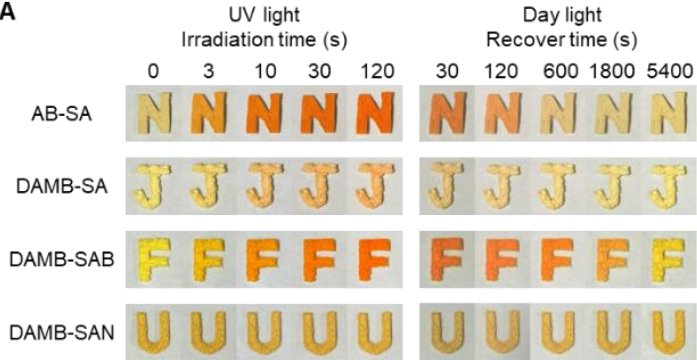

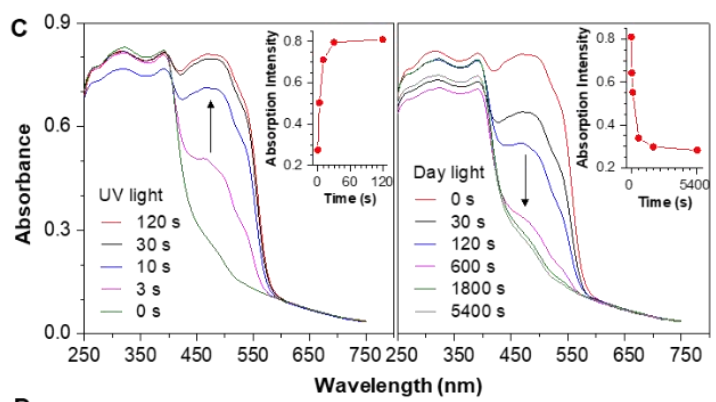

D

B
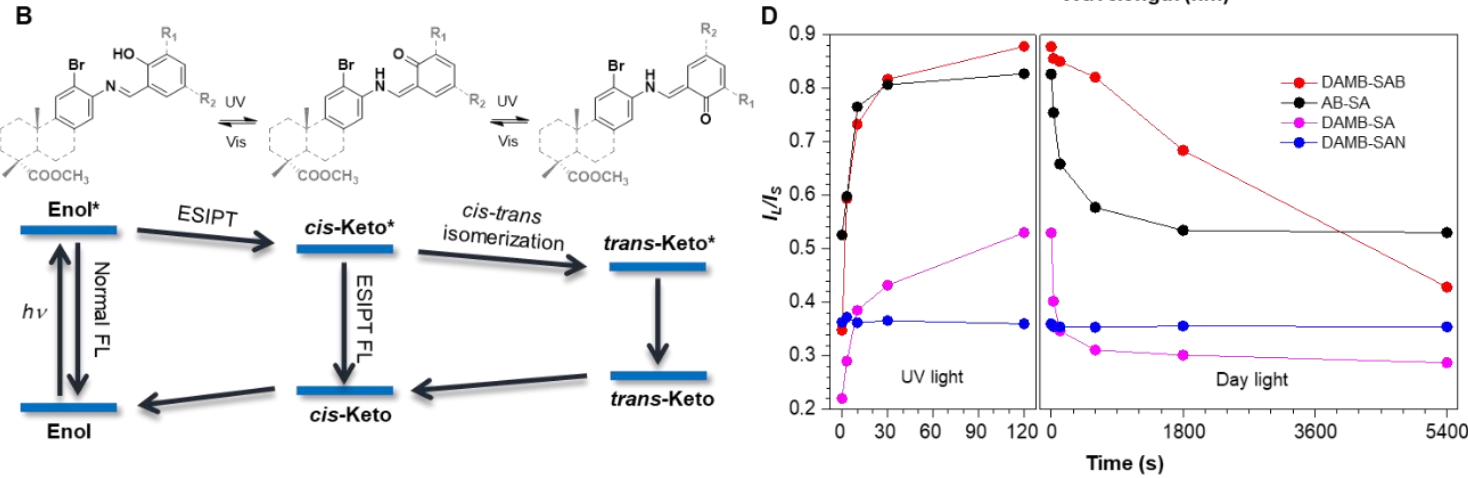

Fig. 4 (A) Photochromic images and (B) the proposed reversible photochromic mechanism of AB-SA, DAMB-SA, DAMB-SAB, and DAMB-SAN upon UV and Day light irradiation. (C) The UV-DRS spectra of AB-SA in its reversible photochromic processes. Inset: The plots of the visible absorption maximum of AB-SA (469 nm) versus the irradiation and recover time, respectively. (D) The plots of the $L_{L} / I_{S}$ versus the irradiation and recover time, respectively. $L$ and $I_{s}$ represent the absorption intensity at the long and short wavelength in the visible region, respectively.

enol to keto under UV light by infrared absorption (Fig. S20), the photochromic property of these BioAlEgens makes it possible to visualize the solid-state molecular motion through color changes. As shown in Fig. 4A, AB-SA exhibits the fastest color darkening at room temperature under UV lightening while this procedure is extremely slowed down at $77 \mathrm{~K}$ (Fig. S21), vividly demonstrating its fast excited-state molecular motion at room temperature. This can be spectrally verified by the fast change in the absorption spectra of AB-SA and its inset (Fig. 4C) in UV lightening process. When it is fused on an alicyclic unit (DAMB-SA), its ambient solidstate molecular motion has been significantly retarded, which can be seen from the much slower color change, suggesting the obvious rigidification effect of the alicyclic moiety. When the bulky $t$-butyl is bis-substituted on the salicyl part (DAMB-SAB), the color change under UV illumination turns faster again, informing us that the bulky and flexible substituent might paly an anti-rigidification role in the excited state, resulting in its faster molecular motion. Specifically, if a rigid nitro group substitutes, barely no color change can be observed in DAMB-SAN because of its scarce molecular motion characteristic. Such color change variations of all alicycle-fused BioAIEgens can be spectrally found in Fig. S22, showing their color darkening sequence completely the same to their molecular motion behaviors. To gain a deeper understanding on the molecular motion behaviors, the fluorescence figures shoot at varied temperatures are shown in Fig. S23. Both AB-SA and DAMB-SAN show high contrast of the emission intensity at RT and $77 \mathrm{~K}$, while the emission contrast of DAMB-SA and DAMBSAN is smaller, indicating that both AB-SA and DAMB-SAB has a dynamic molecular motion at RT, resulting in the excited-state energy loss as non-radiative decay. Similarly, the solid-state Xray diffraction patterns have confirmed this conclusion. As shown in Fig. S24, both AB-SA and DAMB-SAB exhibit nearly identical PXRD patterns as pristine and ground, while DAMB-SA and
DAMB-SAN show distinct PXRD curves, indicating that during the time between grinding and immediate sample measuring, $A B-S A$ and DAMB-SAB are already recrystallized. ${ }^{47}$ As a result, AB-SA and DAMB-SAB have a faster molecular motion compared to DAMB-SA and DAMB-SAN. In addition, the decolorization phenomena in the recover process match well with their absorption spectra. Furthermore, the plots of $I_{L} / I_{S}\left(I_{L}\right.$ and $I_{S}$ are responsible for trans- and cis-Keto absorption, respectively) versus the photochromic time (Fig. 4D), rightly depict the reversible photochromic procedures in Fig. 4A, demonstrating that trans-Keto overwhelms cis-Keto in cases of $A B-S A$ and DAMB-SAB under UV light. The reason why DAMB-SAB has a slower turn-back procedure might be because the bulky $t$-butyl groups could restrain its trans- to cis-Keto transformation at ambient condition. Based on their fundamental structure of $A B$ $S A$, it is reasonable to assume their reversible phtochromic procedure as shown in Fig. 4B, where an excited-state molecular motion occurs between the cis- and trans-Keto forms ${ }^{44-45}$ Undoubtedly, the introduction of the alicyclic structure together with substituent variation could easily regulate their excited-state molecular motion behavior that can be visualize by the photochromic rate from AB-SA, DAMB-SAB, DAMB-SA, to DAMB-SAN.

Targeted imaging. Since the alicyclic moiety originated from natural rosin may endow these BioAlEgens biocompatibility, we thus evaluated the cell and bacterial viability by MTT experiment. As shown in Figs. S25 and S26, more than 90\% viability in the COS-7 cell and $S$. epidermidis and $E$. coli could be remained even at high sample concentrations of $20 \mu \mathrm{M}$, suggesting the excellent cellular biocompatibility of these BioAIEgens. Hence, we have explored the application of these BioAIEgens in COS-7 cell imaging, and the results indicate that DAMB-SA can specifically 


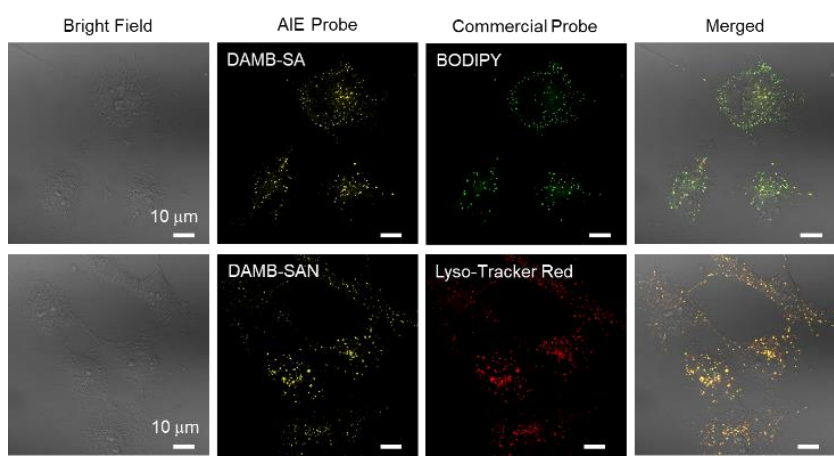

Fig. 5 CLSM images of COS-7 cells stained with DAMB-SA $(10 \mu \mathrm{M})$, BODIPY $(1 \mu \mathrm{M})$, DAMB-SAN $(10 \mu \mathrm{M})$, and Lyso-Tracker Red $(1 \mu \mathrm{M})$, respectively. DAMB-SA channel: $\lambda_{\text {ex: }} 405 \mathrm{~nm}$, $\lambda_{\text {em }}: 540-600 \mathrm{~nm}$; BODIPY channel: $\lambda_{\text {ex: }} 488$ $\mathrm{nm}, \lambda_{\mathrm{em}}$ : 500-530 nm; DAMB-SAN channel: $\lambda_{\text {ex: }} 405 \mathrm{~nm}$, $\lambda_{\mathrm{em}}: 540-600 \mathrm{~nm}$; Lyso-Tracker Red channel: $\lambda_{\mathrm{ex}}: 543 \mathrm{~nm}, \lambda_{\mathrm{em}}: 580-650 \mathrm{~nm}$

stain lipid droplets (LDs), while DAMB-SAN prefers to stain lysosome (Fig. 5). The LDs selectivity of DAMB-SA is possibly ascribed to the lipotropy of its alicyclic moiety. On the other hand, the nitro group in DAMB-SAN is acidophilic, ${ }^{52}$ therefore preferring to enter the slightly acidic lysosome. In addition, the bacterial imaging (Fig. S26A and C) indicates that DAMB-SA and DAMB-SAN can stain both Gram-positive ( $S$. epidermidis) and Gram-negative ( $E$. coll) bacteria. Such results have verified the universality of our BioAIEgens in bio-application. As a key factor of bioimaging, the photostability tests of these DAMB-SA and DAMB-SAN in COS-7 cells are also provided (Fig. S27). The fluorescence signals of the BioAlEgens slightly decrease to $\sim 98 \%$ of their initial values, and the morphology of the cells is still clear after one-photon laser irradiation. In comparison, the fluorescence signals of BODIPY and Lyso-Tracker Red fade to $\sim 73 \%$ and $\sim 87 \%$ of their initial values during the same process, respectively. As a result, the BioAlEgens exhibit superior photostability, which is favorable for the bioimaging.

\section{Discussion}

Natural product-derived compounds are one of the most significant bioactive nature-based compounds due to their natural availability, renewability, sustainability, biodegradability, and biocompatibility compared to the pure artificial chemicals. ${ }^{22}$ Among them, rosin is a renewable and sustainable natural resource, which is nevertheless limited to low value-added applications in current industry. In order to add high values of the rosin-derived biomaterials, we have herein succeeded a new class of BioAlEgens from the abundant and low-cost natural rosin via facile Schiff base reactions. As expected, the introduction of the naturally alicyclic moiety favors the rigidification of the flexible $A B-S A$ and suppression of excitedstate molecular motion, hence endowing it with AIE characteristic. Furthermore, the substituent variation makes it possible to regulate their molecular motion behaviors. Such molecular motion discrepancies can be visualized by distinct photophysical properties: the ones with dynamic molecular motion are inclined to weaker solid-state emission and stronger photochromism, while those with restricted molecular motion prefers stronger solid-state emission and weaker photochromism. These BioAIEgens could respectively target lipid droplet and lysosome with high biocompatibility. Therefore, this work not only provides a new design hypothesis of AIEgens, but also endows the natural rosin with higher added-values.

\section{Methods}

Crystal growth. Only rough crystallographic data can be found in the literature for $A B-S A^{42}$, hence, its single crystal suitable for $X$-ray crystallographic measurements was cultured, remeasured, resolved, and refined, in order to meet the discussion requirements in this work. Single crystals suitable for X-ray crystallographic measurements were respectively obtained by slow evaporation from the EtOH solutions for $A B-S A$ and DAMB$\mathrm{SA}$, from a $\mathrm{ACN} / \mathrm{CH}_{2} \mathrm{Cl}_{2}(5: 6, \mathrm{v} / \mathrm{v})$ solution for DAMB-SAB, and from a $\mathrm{EtOH} / \mathrm{CH}_{2} \mathrm{Cl}_{2}(1: 1, \mathrm{v} / \mathrm{v})$ mixture for DAMB-SAN.

\section{Data availability}

The authors declare that all the data supporting the findings of this manuscript are available within the manuscript and Supporting Information files and also available from the corresponding authors upon reasonable request. CCDC numbers of 1974101, 1963938, 1963939, and 2019547 contain the crystallographic data for AB-SA, DAMB-SA, DAMB-SAB, and DAMB-SAN in this work. These data can be obtained free of charge from the Cambridge Crystallographic Data Centre via www.ccdc.cam.ac.uk/data_request/cif. Source data are provided with this paper.

\section{References.}

1 D'Andrade, B. Lighting: White phosphorescent LEDs offer efficient answer. Nat. Photonics 1, 33-34 (2007).

2 Poletti, F. et al. Towards high-capacity fibre-optic communications at the speed of light in vacuum. Nat. Photonics 7, 279-284 (2013).

3 Huang, B., Wang, W., Bates, M. \& Zhuang, X. Threedimensional super-resolution imaging by stochastic optical reconstruction microscopy. Science 319, 810-813 (2008).

4 Safford, W. E. Lignum Nephriticum-Its History and an Account of the Remarkable Fluorescence of Its Infusion. 271-298 (Ann. Rep. Smithsonian Inst., 1915).

5 Acuña, A. U. More Thoughts on the Narra Tree Fluorescence. J. Chem. Educ. 84, 231 (2007).

6 Valeur, B. \& Berberan-Santos, M. N. A Brief History of Fluorescence and Phosphorescence before the Emergence of Quantum Theory. J. Chem. Educ. 88, 731-738 (2011).

7 Shimomura, O., Johnson, F. H. \& Saiga, Y. Extraction, Purification and Properties of Aequorin, a Bioluminescent Protein from the Luminous Hydromedusan, Aequorea. J. Cell. Comp. Physiol. 59, 223-239 (1962).

8 Weiss, P. S. 2008 Nobel Prize in Chemistry: Green Fluorescent Protein, Its Variants and Implications. ACS Nano 2, 1977-1977 (2008).

9 Roda, A. et al. Chemiluminescence and Bioluminescence: Past, Present and Future. 1-50 (Royal Society of Chemistry, 2011).

10 Kenry, Chen, C. \& Liu, B. Enhancing the performance of pure organic room-temperature phosphorescent luminophores. Nat. Commun. 10, 2111 (2019).

11 Huang, J. et al. Highly Efficient Nondoped OLEDs with Negligible Efficiency Roll-Off Fabricated from AggregationInduced Delayed Fluorescence Luminogens. Angew. Chem. Int. Ed. 56, 12971-12976 (2017). 
$12 \mathrm{Nie}, \mathrm{H}$. et al. Tetraphenylfuran: aggregation-induced emission or aggregation-caused quenching. Mater. Chem. Front. 1, 1125-1129 (2017).

13 Watson, W. F. \& Livingston, R. Concentration Quenching of Fluorescence in Chlorophyll Solutions. Nature 162, 452-453 (1948).

14 Weiss, J. Fluorescence of Organic Molecules*. Nature 152 , 176-178 (1943).

15 Luo, J. et al. Aggregation-induced emission of 1-methyl1,2,3,4,5-pentaphenylsilole. Chem. Commun. 1740-1741 (2001).

16 Chen, Y., Lam, J. W. Y., Kwok, R. T. K., Liu, B. \& Tang, B. Z. Aggregation-induced emission: fundamental understanding and future developments. Mater. Horiz. 6, 428-433 (2019).

$17 \mathrm{He}, \mathrm{X}$. et al. AlE-based theranostic systems for detection and killing of pathogens. Theranostics 9, 3223-3248 (2019).

18 Mei, J., Leung, N. L. C., Kwok, R. T. K., Lam, J. W. Y. \& Tang, B. Z. Aggregation-Induced Emission: Together We Shine, United We Soar. Chem. Rev. 115, 11718-11940 (2015).

19 Zhang, $H$. et al. Clusterization-triggered emission: Uncommon luminescence from common materials. Mater. Today 32, 275-292 (2020).

20 Zhu, C., Kwok, R. T. K., Lam, J. W. Y. \& Tang, B. Z. Aggregation-Induced Emission: A Trailblazing Journey to the Field of Biomedicine. ACS Appl. Bio Mater. 1, 1768-1786 (2018).

21 Zhao, Z., Zhang, H., Lam, J. W. Y. \& Tang, B. Z. AggregationInduced Emission: New Vistas at the Aggregate Level. Angew. Chem. Int. Ed. 59, 9888-9907 (2020).

22 Naghdi, T. et al. Phytochemicals toward Green (Bio)sensing. ACS Sens. 5, 3770-3805 (2020).

$23 \mathrm{He}, \mathrm{T}$. et al. Novel Quercetin Aggregation-Induced Emission Luminogen (AlEgen) with Excited-State Intramolecular Proton Transfer for In Vivo Bioimaging. Adv. Funct. Mater. 28, 1706196 (2018).

$24 \mathrm{Li}$, B. et al. Tumor Inhibition Achieved by Targeting and Regulating Multiple Key Elements in EGFR Signaling Pathway Using a Self-Assembled Nanoprodrug. Adv. Funct. Mater. 28, 1800692 (2018).

25 Long, R. et al. Novel natural myricetin with AIE and ESIPT characteristics for selective detection and imaging of superoxide anions in vitro and in vivo. Chem. Commun. 55, 10912-10915 (2019).

$26 \mathrm{Gu}, \mathrm{Y}$. et al. Visualizing semipermeability of the cell membrane using a $\mathrm{pH}$-responsive ratiometric AlEgen. Chem. Sci. 11, 5753-5758 (2020).

$27 \mathrm{Gu}$, Y. et al. Exploration of biocompatible AlEgens from natural resources. Chem. Sci. 9, 6497-6502 (2018).

$28 \mathrm{Bu}, \mathrm{F}$. et al. Unusual Aggregation-Induced Emission of a Coumarin Derivative as a Result of the Restriction of an Intramolecular Twisting Motion. Angew. Chem. Int. Ed. 54, 14492-14497 (2015).

29 Zhang, Y. et al. Cell Membrane-Specific Fluorescent Probe Featuring Dual and Aggregation-Induced Emissions. ACS Appl. Mater. Interfaces 12, 20172-20179 (2020).

30 Dou, $X$. et al. Clustering-Triggered Emission and Persistent Room Temperature Phosphorescence of Sodium Alginate. Biomacromolecules 19, 2014-2022 (2018).

31 Wang, Q. et al. Reevaluating Protein Photoluminescence: Remarkable Visible Luminescence upon Concentration and Insight into the Emission Mechanism. Angew. Chem. Int. Ed. 58, 12667-12673 (2019).
$32 \mathrm{Ge}, \mathrm{M}$. et al. Seeking brightness from nature: Sustainable carbon dots-based AlEgens with tunable emission wavelength from natural rosin. Chem. Eng. J. https://doi.org/10.1016/j.cej.2020.127457 (2020).

33 Clayton, A. M. et al. Epoxy resins: chemistry and technology. 2nd edn, (Taylor \& Francis, 1985).

34 Zhang, J. et al. Rosin-based Chemicals and Polymers. (Smithers Rapra Technology Ltd, 2012).

35 Zuo, S., Li, S., Zhang, L. \& Luo, J. Chemistry and Technology of Forest Products. 36-42 (China Forestry Publishing House, Beijing, 2019).

36 Zhao, F. et al. Synthesis and potential antineoplastic activity of dehydroabietylamine imidazole derivatives. Med. Chem. Commun. 9, 2091-2099 (2018).

37 Zhao, F. et al. High anticancer potency on tumor cells of dehydroabietylamine Schiff-base derivatives and a copper(II) complex. Eur. J. Med. Chem. 146, 451-459 (2018).

$38 \mathrm{Gu}, \mathrm{W}$. et al. Synthesis and in vitro cytotoxic evaluation of new $1 \mathrm{H}$-benzo[d]imidazole derivatives of dehydroabietic acid. Bioorg. Med. Chem. Lett. 27, 1296-1300 (2017).

39 Bernardo, G., Esteves, M. A., Guerreiro, A. M., Gigante, B. \& Morgado, J. Luminescence properties of bipolar stylbeneamine-quinoxalines. Opt. Mater. 31, 320-327 (2008).

40 Gao, H., Chen, S., Rao, X., Shang, S. \& Song, Z. A new dehydroabietic acid-based arylamine fluorescent probe: Synthesis, structure analysis and in vitro biodiagnose function. Bioorg. Med. Chem. Lett. 23, 2254-2259 (2013).

41 Cai, X.-M. et al. Syntheses, Crystal Structures, and Electrochemical Properties of Dehydroabietic Acid-based Schiff Bases. Chinese J. Struct. Chem. 39, 1699-1706 (2020).

42 Burr, A. H. \& Hobson, A. D. The crystal and molecular structure of 2-bromo-N-salieylideneaniline. Acta Cryst. B25, 2662-2663 (1969).

43 Barbara, P. F., Rentzepis, P. M. \& Brus, L. E. Photochemical Kinetics of Salicylidenaniline. J. Am. Chem. Soc. 102, 27862791 (1980).

44 Sun, $H$. et al. A new tetraphenylethene-based Schiff base: two crystalline polymorphs exhibiting totally different photochromic and fluorescence properties. J. Mater. Chem. C 7, 7053-7060 (2019).

45 Wang, L. et al. An erasable photo-patterning material based on a specially designed 4-(1,2,2-triphenylvinyl)aniline salicylaldehyde hydrazone aggregation-induced emission (AIE) molecule. J. Mater. Chem. C 5, 65-72 (2017).

46 Chakraborty, S. et al. How cholesterol stiffens unsaturated lipid membranes. Proc. Natl. Acad. Sci. USA 117, 2189621905 (2020)

47 Alam, P. et al. Spontaneous and Fast Molecular Motion at Room Temperature in the Solid State. Angew. Chem. Int. Ed. 58, 4536-4540 (2019).

48 Liu, S. et al. Molecular Motion in the Solid State. ACS Materials Lett. 1, 425-431 (2019).

49 Zhang, J. et al. Molecular Motions in AlEgen Crystals: Turning on Photoluminescence by Force-Induced Filament Sliding. J. Am. Chem. Soc. 142, 14608-14618 (2020).

50 Yang, Y.-D. et al. Time-dependent solid-state molecular motion and colour tuning of host-guest systems by organic solvents. Nat. Commun. 11, 77 (2020).

51 Zhang, $\mathrm{H}$. et al. Visualization and Manipulation of Molecular Motion in the Solid State through Photoinduced 
Clusteroluminescence. J. Phys. Chem. Lett. 10, 7077-7085 (2019).

52 Fita, P., Fedoseeva, M. \& Vauthey, E. Ultrafast Excited-State Dynamics of Eosin B: A Potential Probe of the HydrogenBonding Properties of the Environment. J. Phys. Chem. A 115, 2465-2470 (2011).

\section{Acknowledgements}

Support from the National Natural Science Foundation of China (21601087 and 21805233) is gratefully acknowledged. We also thank the Science and Technology Plan of Shenzhen (JCYJ20180306174910791) and the Innovation and Technology Commission (ITC-CNERC14SC01 and ITCPD/179). The authors also acknowledge the Advanced Analysis \& Testing Center of Nanjing Forestry University for testing services.

\section{Author contributions}

X.-M.C., Z.Z., and B.Z.T. conceived and designed the experiments. Y.L. (Yuting Lin) and X.C. performed the synthesis. X.-M.C., Y.L. (Yuting Lin), and X.C. did the PL measurements and analyzed the data. Z.W. and X.Z. conducted QY measurements. Y.L. (Ying Li) performed the cell culturing and imaging tests as well as cytotoxicity experiments. Z.Z. performed the theoretical calculation. X.-M.C., S.H., Z.Z., and B.Z.T. took part in the discussion and give important suggestions. X.-M.C., and Z.Z., co-wrote the paper.

\section{Additional information}

Supporting Information accompanies this manuscript can be found online.

Competing interests: The authors declare no competing interests. 